\title{
Noninterleaved round beam lattice for light sources
}

\author{
Ilya Agapov, Reinhard Brinkmann, Joachim Keil, and Rainer Wanzenberg \\ DESY, Notkestrasse 85, 22607 Hamburg, Germany
}

(Received 16 March 2018; published 29 May 2018)

\begin{abstract}
A conceptual design and performance of a round beam lattice for synchrotron light sources based on the phase space exchange principle and the noninterleaved sextupole distribution is presented. Optics design is performed for an approximately $30 \mathrm{pm}$ emittance $6 \mathrm{GeV}$ machine of $2300 \mathrm{~m}$ circumference which combines cells with and without straight sections for the insertion devices.
\end{abstract}

DOI: 10.1103/PhysRevAccelBeams.21.051601

\section{INTRODUCTION}

Synchrotron light sources operate with flat beams, but round beams might be desirable for mitigating the effect of intrabeam scattering (IBS) [1,2] or for improving the optical properties of the produced undulator radiation [3]. There are several approaches to producing round beams in synchrotrons [4], the usual one consisting in exploiting the natural coupling of the machine. The coupling is typically a small undesired effect in a synchrotron and has to be amplified by working on a resonance [5]. Resonances are by definition areas of sensitive and irregular motion. Small changes to the tune could strongly affect the emittance; optics measurement and correction can be extremely nontrivial. Thus, another approach consists in introducing full coupling nonresonantly. Working with a fully coupled optics is difficult and multiple parametrizations of the optical functions are in use (see e.g. [6-9]). A simple way to introduce full coupling is to flip the horizontal and vertical modes of betatron oscillation a finite number of times in the ring so that on average the number of oscillations in both planes is roughly equal; the coupling in this case is localized and affects only integral characteristics such as the emittance. One does not need to resort to coupled beam envelope formalism for optics design, matching etc. It is technically simplest to implement just one mode flip per revolution; the concept has been studied for round beam production both theoretically [10-13] and experimentally [14] and is known as the "Möbius accelerator." A round beam lattice with two flips is employed for the VEPP2000 collider [15] for reasons of improving the luminosity.

The new generation of synchrotron light sources [16-19] push down the beam emittance by employing the multibend

Published by the American Physical Society under the terms of the Creative Commons Attribution 4.0 International license. Further distribution of this work must maintain attribution to the author(s) and the published article's title, journal citation, and DOI. achromat (MBA) concept [20-24]. In such lattices strong focusing produces large negative chromaticity, and the sextupoles needed to compensate it are typically strong. This, in turn, has a strong impact on the dynamic aperture and the momentum acceptance. A usual path to improving the dynamic aperture is to arrange sextupoles so that their detrimental effects cancel to the highest possible order. One of the simplest schemes to do so is to arrange for a phase advance of $\pi$ in both planes between adjacent sextupoles of same polarity. Such a scheme is referred to as the noninterleaved scheme. The nonlinear effects of sextupoles in the thin-lense on-momentum approximation cancel, and a very large dynamic aperture is possible $[25,26]$.

In this paper we explore the combination of the two approaches-phase space flip and the noninterleaved sextupole scheme-for the design of a synchrotron light source lattice. The optics approach and conceptual lattice design for the PETRA ring [27] upgrade based on this scheme is presented in Sec. II. Nonlinear properties of the noninterleaved optics and a study of resonances in a simplified model of the phase space exchange lattice are presented in Sec. III. After that, the acceptance of the lattice is presented in Sec. IV.

The parameters of the lattice presented here are such that it is a possible lattice for the upgrade of the PETRA III light source at DESY [27-29]. Several lattice types are under consideration, and the lattice discussed here falls into a more conservative category with about $30 \mathrm{pm}$ rad bare lattice emittance. More aggressive designs with down to $10 \mathrm{pm}$ rad emittance are also being considered, and the lattice selection process in currently ongoing. The projected parameters for PETRA IV are shown in Table I.

\section{LATTICE}

\section{A. Principles}

The first principle exploited in the presented lattice is using different cell types in different octants. PETRA III has been converted into a light source from an originally 
TABLE I. Target PETRA IV parameters.

\begin{tabular}{lc}
\hline \hline Parameter & PETRA IV \\
\hline Beam energy & $6 \mathrm{GeV}$ \\
Circumference & $2304 \mathrm{~m}$ \\
No. bunches & $80-4000$ \\
Emittance $\varepsilon_{x}, \varepsilon_{y}$ & $10-30,10-30 \mathrm{pm} \mathrm{rad}$ \\
Energy spread & $0.7-1.5 \times 10^{-3}(4.2-9 \mathrm{MeV})$ \\
Bunch length & $10-50 \mathrm{ps}(\mathrm{rms})$ \\
Beam current & $100-200 \mathrm{~mA}$ \\
$\beta$-function at IDs & $1-7 \mathrm{~m}$ \\
\hline \hline
\end{tabular}

high energy physics collider ring, which defines its geometry: eight octants linked by eight straight sections of approximately 100 or $60 \mathrm{~m}$ length, with only one octant initially occupied by the double-bend achromats (DBAs), and the rest by FODO cells without the insertion devices. Parts of two additional octants were converted to DBA optics during an extension project [28]. The proposed facility layout for the next upgrade is shown in Fig. 1. The specific geometry can be made use of in the lattice design. In the lattice presented here, two octants are occupied by cells with insertion devices, and the rest of the ring has optics-long achromats-where no place for insertions is foreseen.

The second principle is the noninterleaved sextupole arrangement, mentioned before. Finally, two phase space exchange sections make it possible to use only horizontally focusing sextupoles to correct the chromaticity in both planes simultaneously. If we call the parts of the ring between the phase space exchange sections $A$ and $B$, then correcting the ring to zero chromaticity requires

$$
\xi_{x}^{A}+\xi_{y}^{B}=0 \quad \xi_{y}^{A}+\xi_{x}^{B}=0 .
$$

The chromaticity correction can be distributed over various cell types, as discussed further. The phase space exchange can be installed in any of the long straight sections, one possible choice is indicated in Fig. 1 with arrows. The lattice on which the calculations are based has periodicity two, with one undulator octant and three long achromat octants per half of the ring.

\section{B. Noninterleaved undulator cells}

An optics solution employed at all modern light sources that minimizes the emittance [30,31] while providing zero (or close to zero) dispersion is the multibend achromat (MBA). A $23 \mathrm{~m}$ long six bend achromat (6BA) cell, with $\pi$ phase advance between the two focusing sextupoles, is used in the present lattice. The cell optical functions are shown in Fig. 2. An arc consists of nine such cells. The cell phase advances are chosen such that the phase advances per arc are integer multiples of $2 \pi$ in both planes. The chromaticities of the cell depending on the strength of the focusing sextupole pair are shown in Table II.

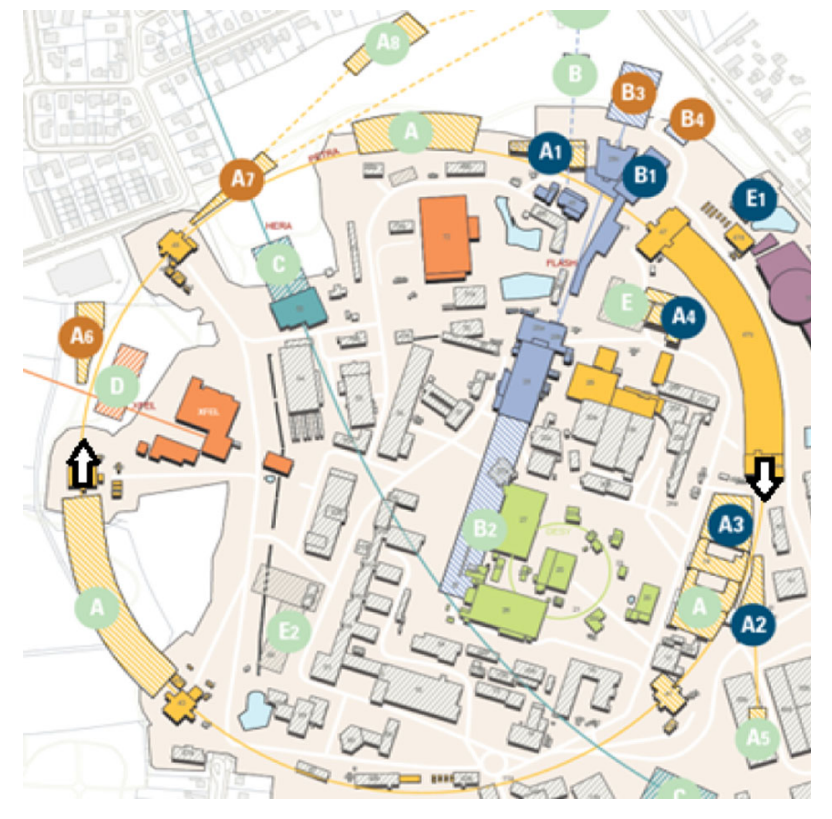

FIG. 1. Layout of the PETRA III/IV infrastructure. Positions of the phase space exchange sections following the two octants with insertion devices are shown with arrows.

\section{Long achromats}

In the arcs without the insertion devices, a simpler optics is possible. The arc is made of $1512 \mathrm{~m}$ long unit cells and two dispersion suppressor cells at the arc ends. The optical functions of the cell are shown in Fig. 3. The lattice is a modification of a 6BA cell with vertically focusing combined function magnets, a common approach in lowemittance lattices. Strong defocusing in combined-function magnets improves the emittance [32], but can potentially lead to the longitudinal partition shifting towards zero, resulting in a large natural energy spread or even longitudinal instability. In order to reasonably balance the

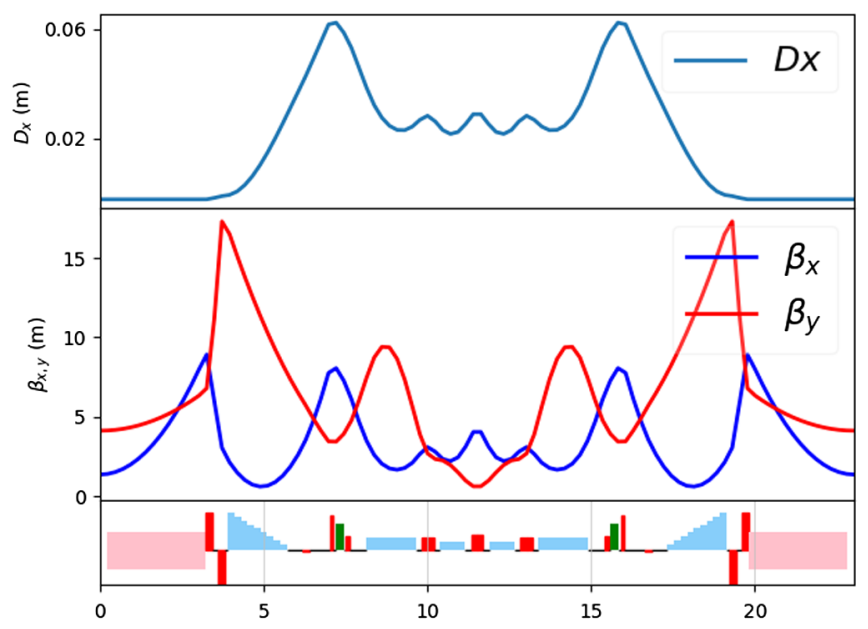

FIG. 2. Possible cell optics for the insertion. The four central bends are combined-function magnets. 
TABLE II. Cell chromaticities vs sextupole strength for the undulator cell (Cell 1) and for the cell in the arcs without insertions (Cell 2).

\begin{tabular}{ccccc}
\hline \hline & Parameter & Natural & $\xi_{x}=0$ & $\xi_{x}+\xi_{y}=0$ \\
\hline Cell 1 & $m_{s}\left(\mathrm{~T} \mathrm{~m}^{-2}\right)$ & 0 & 34 & 85 \\
& $\xi_{x}$ & -2.6 & 0 & 5 \\
& $\xi_{y}$ & -2.4 & -3 & -5 \\
& $\xi_{x}+\xi_{y}$ & -5 & -3 & 0 \\
Cell 2 & $m_{s}\left(\mathrm{~T} \mathrm{~m}^{-2}\right)$ & 0 & 22 & 65 \\
& $\xi_{x}$ & -0.8 & 0 & 1.6 \\
& $\xi_{y}$ & -1.1 & -1.2 & -1.6 \\
& $\xi_{x}+\xi_{y}$ & -1.9 & -1.2 & 0 \\
\hline \hline
\end{tabular}

transverse and longitudinal partition shifts we also introduced three horizontally focusing combined function magnets for better control of the partition number distribution. The optical functions of the octant are shown in Fig. 4. The chromaticities of the cell as a function of the strength of the focusing sextupole pair are shown in Table II.

\section{Phase space exchange}

The long straight sections are realized with triplet optics. In addition two of the straights have a phase space exchange section. It is a $\pi$ phase advance FODO section with the normal quadrupole component changed to skew quadrupole component, which has the $4 \times 4$ transfer matrix

$$
\mathcal{R}=\left[\begin{array}{llll}
0 & 0 & 1 & 0 \\
0 & 0 & 0 & 1 \\
1 & 0 & 0 & 0 \\
0 & 1 & 0 & 0
\end{array}\right] .
$$

The same section has an identity transform when the skew quadrupole components are replaced again by normal

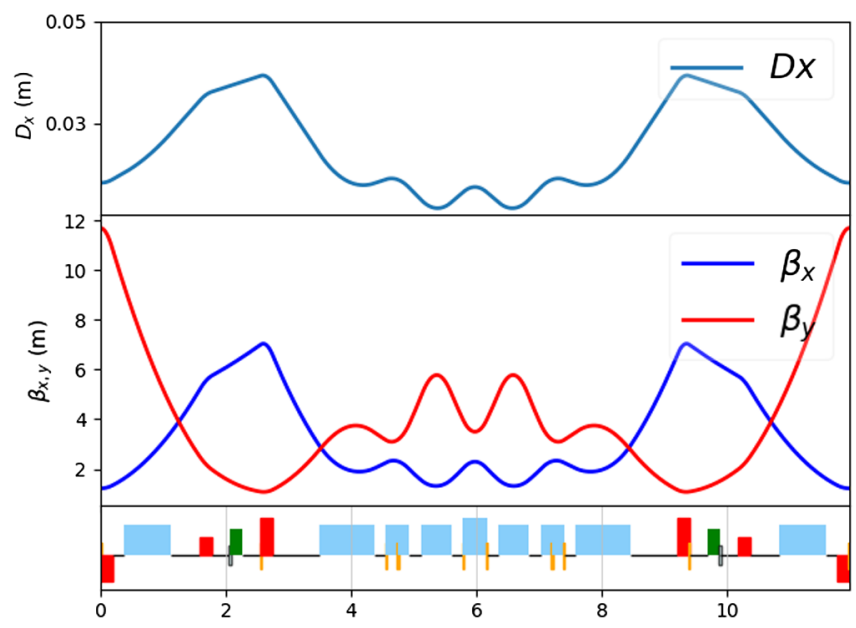

FIG. 3. Optical functions in a cell of the arc (octant) without the undulator insertions.

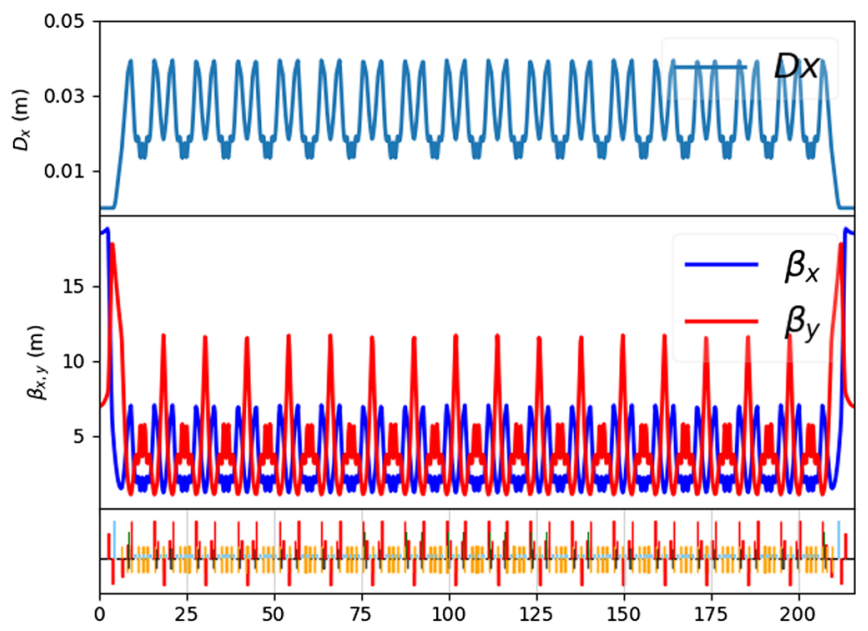

FIG. 4. Optical functions in the arc (octant) without the undulator insertions.

components, e.g. by means of rotation. The optics of such section is shown in Fig. 5. The phase advances of the long straight sections are approximately $2 \pi$, with the fractional phase advance part used for tune matching, and the chromaticities between -2 and -3 units in both planes for all three types of long straight sections.

\section{E. Lattice parameters}

The Mais-Ripken coupled lattice functions [8] of the ring are shown in Fig. 6 (for discussion of coupled lattice functions see e.g. [9]).

The lattice parameters are presented in Table III. Note that the insertion devices have a significant impact on the equilibrium emittance and the energy spread. So, if all (short) straight sections in the two octants are occupied by $23 \mathrm{~mm}$ period undulators with $0.7 \mathrm{~T}$ rms field, the emittance will be reduced by more than a factor of 2 . Also note that the effect of a small longitudinal partition number (approximately 0.1 ) on the energy spread is mitigated by undulators or damping wigglers. This follows from

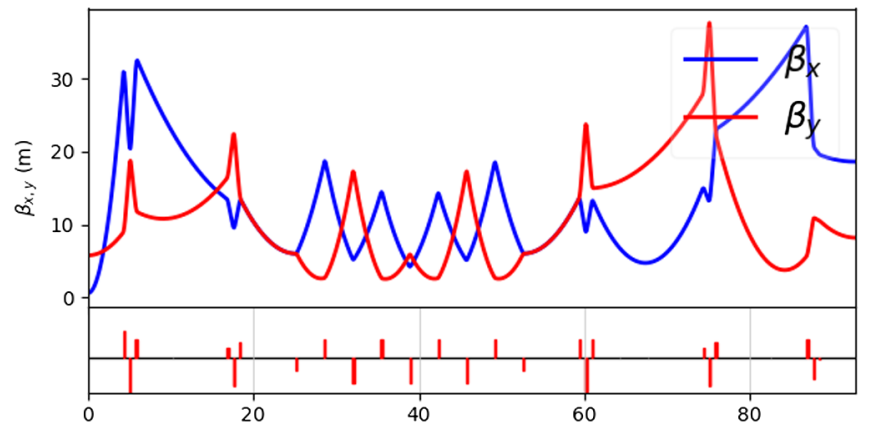

FIG. 5. Straight section with the triplet optics including the skew FODO phase space exchange section. The optical functions are plotted for the quadrupoles set in the normal (not skew) mode. 

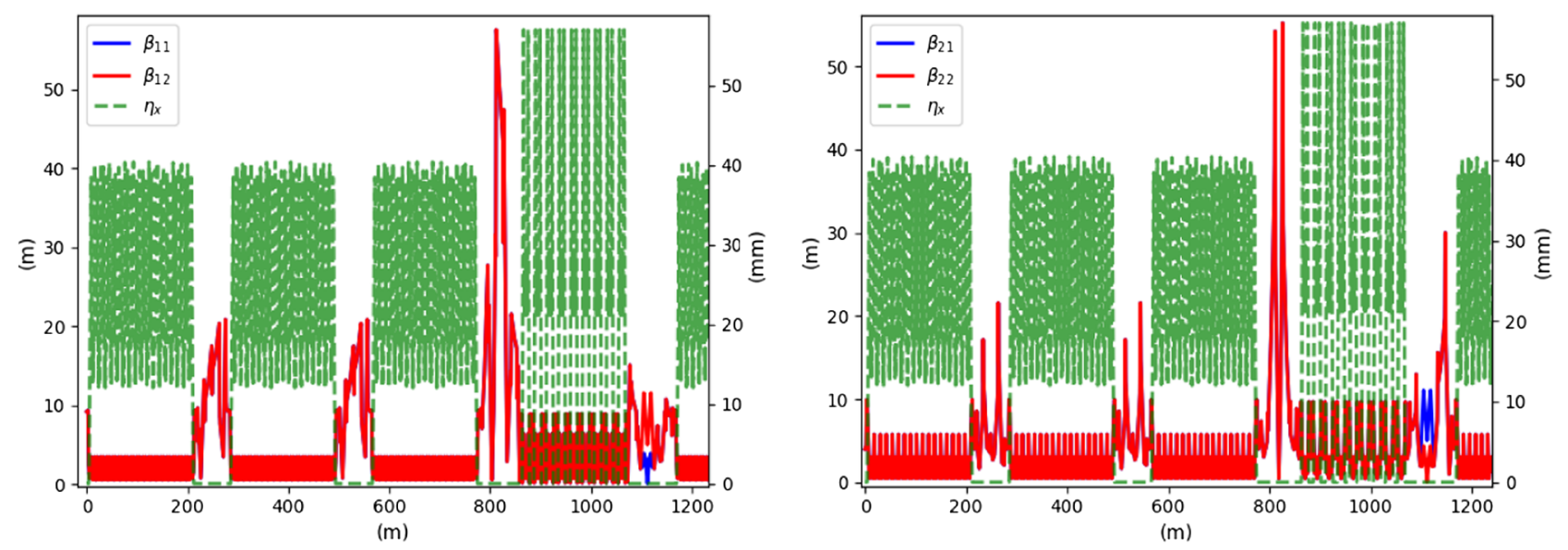

FIG. 6. Mais-Ripken optical functions of the full lattice. Half of the ring (four octants) is shown.

$$
\left(\frac{\sigma_{E, \mathrm{wig}}}{\sigma_{E, 0}}\right)^{2}=\frac{1+\frac{\mathcal{I}_{3, \mathrm{wig}}}{\mathcal{I}_{3,0}}}{1+\frac{\mathcal{I}_{2, \mathrm{wig}}}{\mathcal{I}_{2,0}}} \frac{j_{z, 0}}{j_{z, \mathrm{wig}}},
$$

where $\mathcal{I}_{2}$ and $\mathcal{I}_{3}$ are the radiation integrals and $j_{z}$ the longitudinal partition number defined in the usual way [33], and the subscripts indicate the values with and without damping wigglers.

The question of dynamic aperture and momentum acceptance of the lattice is discussed in the next sections.

\section{NONLINEAR DYNAMICS}

\section{A. Nonlinear dynamics in noninterleaved lattices}

In the noninterleaved optics sextupole kicks separated by $\pi$ phase advance cancel exactly (neglecting the sextupole length effect which is small in practice), and the onmomentum dynamic aperture is large without any need for further nonlinearity cancellation. The higher-order chromaticities, however, are hard to compensate (see e.g. [34]) and become a limiting factor in such lattices. In the lattice presented here, the distribution of sextupole strengths between two types of octants becomes an additional knob for higher-order chromaticity control. In Fig. 7 sums of second-order chromaticities for the two types of cells in the ring are shown. The calculations were done with

TABLE III. Lattice parameters, collective effects neglected.

\begin{tabular}{lc}
\hline \hline Parameter & Value \\
\hline Beam energy & $6 \mathrm{GeV}$ \\
Emittance, bare lattice (horizontal, vertical) & $28 \mathrm{pm}, 28 \mathrm{pm}$ \\
Emittance, 0.7 T (rms) IDs & $12 \mathrm{pm}, 12 \mathrm{pm}$ \\
Energy spread, bare lattice & $2.6 \times 10^{-3}$ \\
Energy spread, 0.7 T IDs & $1.0 \times 10^{-3}$ \\
Integer tunes & 123,123 \\
Natural chromaticity & $-188,-188$ \\
\hline \hline
\end{tabular}

the PTC module integrated with MADX $[35,36]$. In terms of second-order chromaticity cancellation, neither distributing the correction between two types of cells equally, nor having all correction done in the no-undulator cells is optimal. The region chosen for tracking studies in this work is highlighted in red and corresponds to somewhat redistributing the linear chromaticity correction towards the undulator cells, with the sextupole strengths in all cells larger than what is required for cell chromaticity compensation due to the additional contribution of the long straight sections (cf. Table II). Note that the second-order chromaticity of the ring is not a simple sum of cell chromaticities (see [34]), and the higher-order terms play an important role, so the second-order cell analysis gives only a starting point for further optimization.

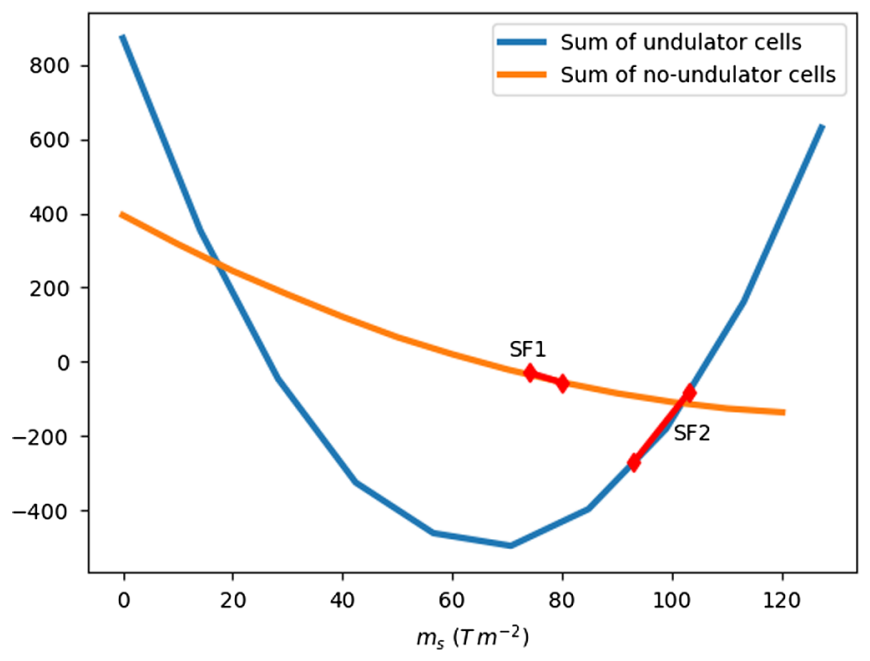

FIG. 7. Sums of second-order chromaticities (horizontal plus vertical) over the total number of cells in the ring. The horizontal axis shows sextupole strength in two different cell types, and the regions highlighted in red correspond to the ranges of strengths for linear chromaticity compensation used in the tracking studies. 


\section{B. Study of stability domain in a simplified phase space exchange model}

Although various nonlinear characteristics of particle motion could be derived perturbatively to lowest orders, they are often represented by entangled expressions and cannot provide a quantitative description of the figures of merit such as the dynamic aperture. The situation becomes even more complicated when coupling is introduced, so to proceed further with the discussion of the effect of phase space exchange on the dynamics, a simplified model is introduced. Following the usual approach, we model a one turn map as a linear map plus a nonlinear kick. In addition, the phase space exchange is introduced. Consider first the sextupole nonlinearity, modeled by the combination of linear, sextupole, and phase space exchange maps applied two times as follows:

$$
\mathcal{M}_{3}=\mathcal{M}_{S} \cdot \mathcal{M}_{T W} \cdot \mathcal{M}_{L I N} \cdot \mathcal{M}_{S} \cdot \mathcal{M}_{T W} \cdot \mathcal{M}_{L I N}
$$

where the linear part is a $4 \times 4$ matrix:

$$
\begin{gathered}
\mathcal{M}_{\text {LIN }}:\left(\begin{array}{cc}
\mathcal{M}_{\text {LIN,X}} & 0 \\
0 & \mathcal{M}_{L I N, Y}
\end{array}\right) \\
\mathcal{M}_{L I N, X, Y}:\left(\begin{array}{cc}
\cos \left(2 \pi \mu_{x, y}\right) & \sin \left(2 \pi \mu_{x, y}\right) \\
-\sin \left(2 \pi \mu_{x, y}\right) & \cos \left(2 \pi \mu_{x, y}\right)
\end{array}\right),
\end{gathered}
$$

the phase space exchange part is a $4 \times 4$ matrix

$$
\mathcal{M}_{T W}:\left(\begin{array}{cc}
0 & \mathcal{I} \\
\mathcal{I} & 0
\end{array}\right)
$$

with $\mathcal{I}$ the identity, and the thin lens sextupole map is

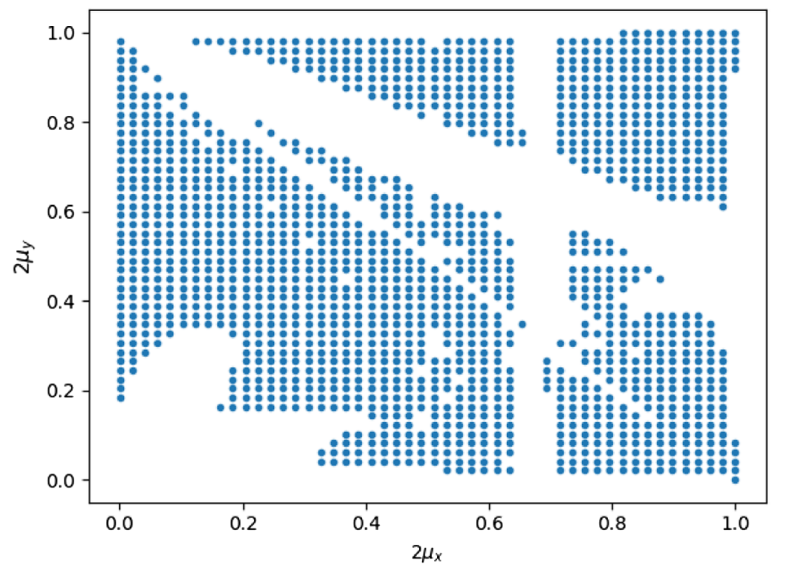

(a) $\tilde{\mathcal{M}}_{3}$

$$
\mathcal{M}_{S}:\left(\begin{array}{c}
x \\
x^{\prime} \\
y \\
y^{\prime}
\end{array}\right) \rightarrow\left(\begin{array}{c}
x \\
x^{\prime}-m\left(x^{2}-y^{2}\right) \\
y \\
y^{\prime}+2 m x y
\end{array}\right) \text {. }
$$

This is a greatly simplified model of a phase space exchange lattice with the tunes $\mu_{x}+\mu_{y}$ and a residual sextupole aberration averaged over half of the ring. The longitudinal degree of freedom is neglected. When the phase space exchange is removed from the map, it becomes a usual toy model of Hénon-Heiles type [37] used in studying nonlinear dynamics in storage rings

$$
\tilde{\mathcal{M}}_{3}=\mathcal{M}_{S} \cdot \mathcal{M}_{L I N} \cdot \mathcal{M}_{S} \cdot \mathcal{M}_{L I N}
$$

We will study the stability of such maps as a function of the phase advances of the linear part. The nonlinearity parameter $m$ is not essential since the oscillation amplitudes can be always rescaled, so $m$ will be arbitrarily fixed to 0.5. In Fig. 8 stability diagrams of the maps $\tilde{\mathcal{M}}_{3}$ and $\mathcal{M}_{3}$ depending on the phase advances are shown. The diagrams are produced as follows: tracking is done for a certain sufficiently large initial $x$ and $y$ for $10^{4}$ turns. The oscillations are either bounded or grow very rapidly. The initial phase advances for which the oscillations are bounded are plotted in the diagram. A sufficiently small initial condition will produce stable motion for all phase advances, while a sufficiently large initial condition will produce a motion which is unstable for all phase advances, so the value $x_{0}=y_{0}=0.5$ is representative of the regime where stable and unstable domains are clearly visible. The effect of taking a different initial condition is mainly quantitative, the stability domain is larger for smaller initial conditions, while the general resonance picture remains. In the uncoupled case the unstable resonances are seen, with integer and third integer being the strongest. In the coupled

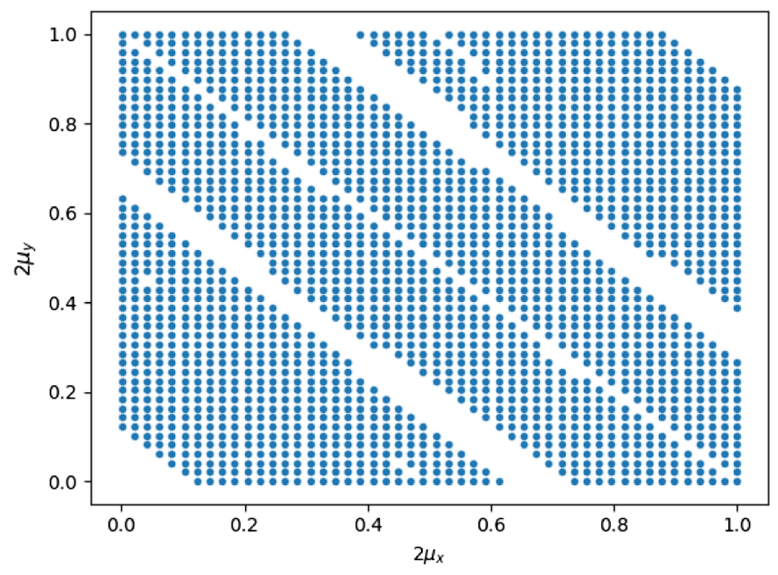

(b) $\mathcal{M}_{3}$

FIG. 8. Stability diagrams of third-order maps, $x_{0}=y_{0}=0.5$. 


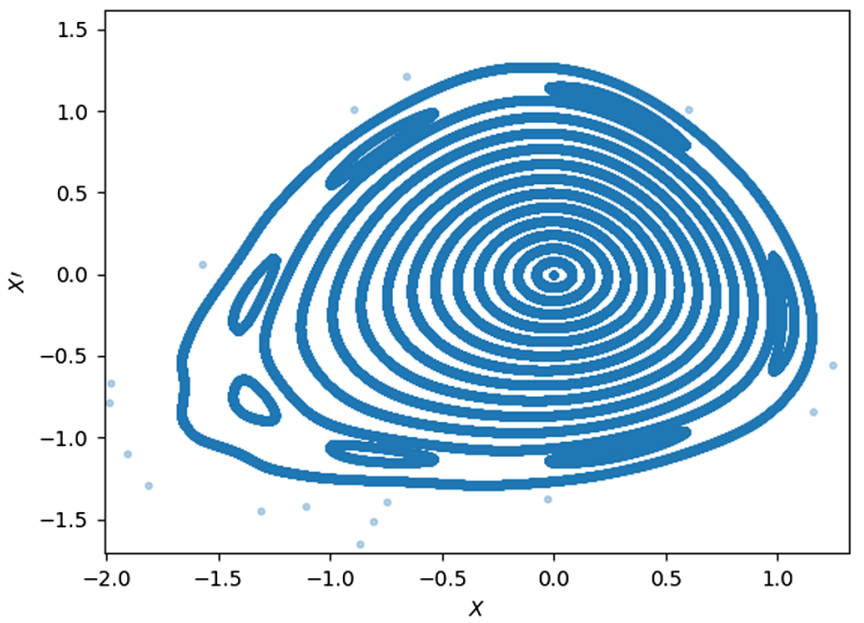

FIG. 9. Phase space portrait of $\tilde{\mathcal{M}}_{3}$ (third-order uncoupled map), $\mu_{x}=\mu_{y}=1 / 6$.

case the diagram becomes almost one dimensional (if the projection on the diagonal is considered), and the instability domains correspond to the resonant conditions $2 \mu_{x}+2 \mu_{y}=$ $2 / 3,2 \mu_{x}+2 \mu_{y}=4 / 3,2 \mu_{x}+2 \mu_{y}=1$ and $2 \mu_{x}+2 \mu_{y}=0$.

Next, some examples of phase space portraits are shown in Figs. 9 and 10. Trajectories with different initial amplitudes in the horizontal direction are shown. While for the uncoupled case one observes the well-known picture of transition to instability with resonant islands and chaotic layers, the geometry of the coupled motion is more complex. A multitude of geometric patterns can be produced in that way.

Fourth-order nonlinearities have a significant impact on beam dynamics, so we proceed to analyze the effect of the octupole nonlinearity with model maps

$$
\mathcal{M}_{4}=\mathcal{M}_{O} \cdot \mathcal{M}_{T W} \cdot \mathcal{M}_{L I N} \cdot \mathcal{M}_{O} \cdot \mathcal{M}_{T W} \cdot \mathcal{M}_{L I N}
$$

and

$$
\tilde{\mathcal{M}}_{4}=\mathcal{M}_{O} \cdot \mathcal{M}_{L I N} \cdot \mathcal{M}_{O} \cdot \mathcal{M}_{L I N}
$$

where

$$
\mathcal{M}_{O}:\left(\begin{array}{c}
x \\
x^{\prime} \\
y \\
y^{\prime}
\end{array}\right) \rightarrow\left(\begin{array}{c}
x \\
x^{\prime}+m_{O}\left(\frac{x^{3}}{3}-x y^{2}\right) \\
y \\
y^{\prime}+m_{O}\left(\frac{y^{3}}{3}-x^{2} y\right)
\end{array}\right)
$$

with $m_{O}$ being the octupole strength, set to 1.0 in calculations. The phase space portraits in this case are shown in Fig. 11. Interestingly, two octupole kicks cancel out over one revolution, and the horizontal dynamics does not couple into vertical. The phase space geometry in the two cases is similar. This can be also inferred from the symmetry of the octupole field with respect to interchanging $x$ and $y$. The stability diagrams in both cases are also similar, and shown in Fig. 12.

To summarize the results from the simplified model, the phase space exchange alters the geometry of the phase space, but does not significantly reduce the regions of stability in the tune and in the amplitude space.

\section{ACCEPTANCE OF THE LATTICE}

Analytical estimates and simplified models provide a qualitative description of lattice behavior and strategies for compensating nonlinear aberrations. Quantitative performance estimates and optimization are always performed with particle tracking, which can be a time and CPU consuming procedure for a machine like PETRA. A procedure involving extensive parameter scans or advanced optimization is inevitable for a technical design of a facility. In this paper we present a conceptual design, and extensive optimization is avoided; the parameters are selected based on principles provided in the previous sections. Moreover, already relatively small alignment

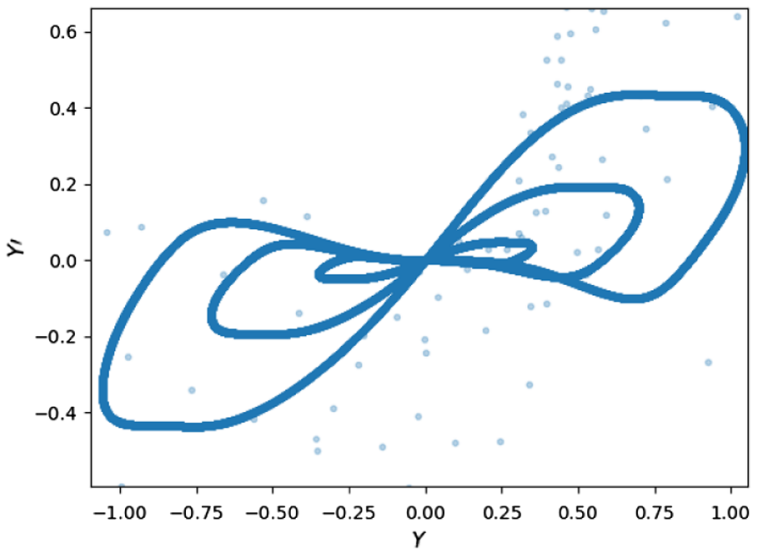

(b) $\mathcal{M}_{3}$

(a) $\mathcal{M}_{3}$

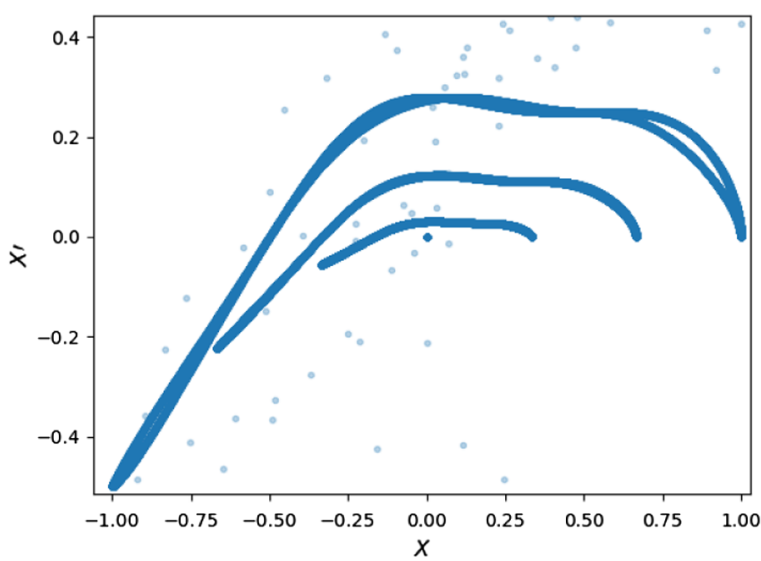

FIG. 10. Phase space portraits of $\mathcal{M}_{3}$ (third-order coupled map), $\mu_{x}=\mu_{y}=1 / 4$. 


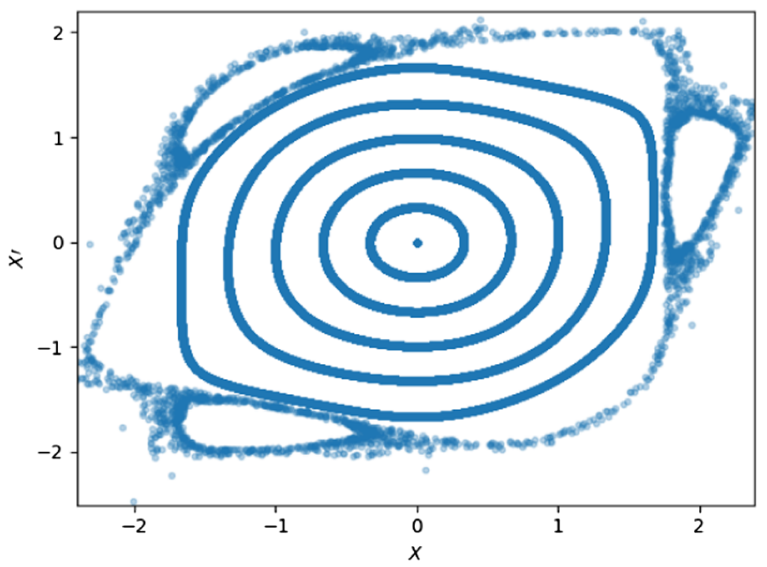

(a) $\tilde{\mathcal{M}}_{4}$

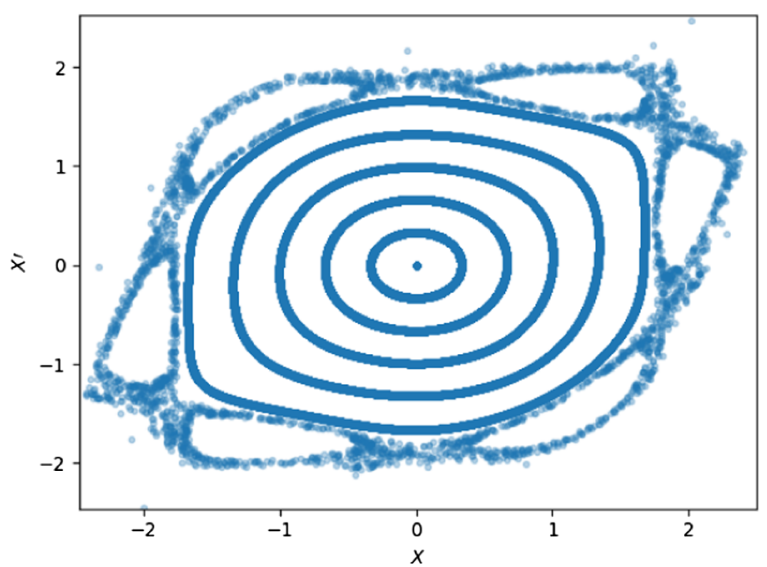

(b) $\mathcal{M}_{4}$

FIG. 11. Phase space portraits of fourth-order maps, $\mu_{x}=\mu_{y}=0.21$.

errors can influence the acceptance of MBA machines. Both the optimization and the tolerance study are subjects of separate work and are not addressed here.

The cell phase advances are chosen to fulfill the achromat condition: the nine undulator cells per arc make the phase advances of 15 and 8 in units of $2 \pi$ in horizontal and vertical planes respectively, while the rest of the ring is such that the horizontal and vertical phase advances of the 17 cells per arc sum up to 13 and 12 . However, the exact fulfillment of this condition is not important for noninterleaved lattices since geometric aberrations are already canceled to a high degree of precision regardless of the cell phase advance. This leaves additional freedom for selecting the beta function in the undulator insertions.

In Fig. 13 the lattice acceptance is shown for fractional tunes $Q_{x}=0.76$ and $Q_{y}=0.76$, and linear chromaticities $\xi_{x}=0$ and $\xi_{y}=0$. A large dynamic aperture (approximately $4.5 \mathrm{~mm}$ mrad or $15 \mathrm{~mm}$ at $\beta_{x}=50 \mathrm{~m}$ horizontally, and approximately $2.5 \mathrm{~mm} \mathrm{mrad}$ or $11 \mathrm{~mm}$ at $\beta_{y}=50 \mathrm{~m}$ vertically) and a momentum acceptance of approximately $1.6 \%$ can be observed. The tune footprint is shown in

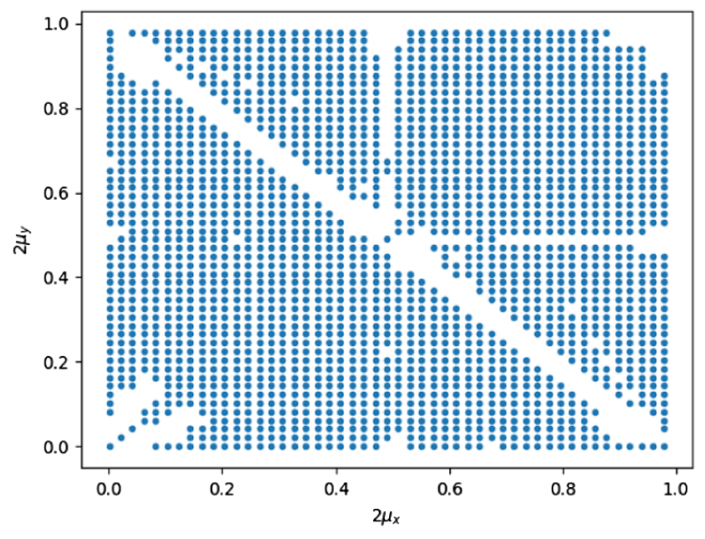

FIG. 12. Stability diagram of $\mathcal{M}_{4}, x_{0}=0.5, y_{0}=0.5, m_{O}=1.0$.
Fig. 14, the integer, $Q_{x}=2 / 3, Q_{y}=2 / 3$ and the $Q_{x}+$ $Q_{y}=4 / 3$ resonances confining the tune footprint.

A known feature of low momentum compaction lattices (the present lattice has $\alpha_{C}=4 \times 10^{-5}$ ) is the strong coupling of transverse oscillations into the longitudinal dimension due to the path lengthening effect. The shift of the path length per revolution is given to first order by $[38,39]$

$$
\Delta C=-2 \pi\left(J_{X} \xi_{x}+J_{Y} \xi_{y}\right),
$$

where $J_{X}$ and $J_{Y}$ are betatron actions, and $\xi_{x}$ and $\xi_{y}$ are the chromaticities. Longitudinal oscillations are thus excited for particles with large transverse offsets, which limits the dynamic aperture. For a $100 \mathrm{MHz}$ rf system with $10 \mathrm{MV}$ total voltage, the limitation is about $2 \mathrm{~mm}$ mrad as shown in Fig. 13.

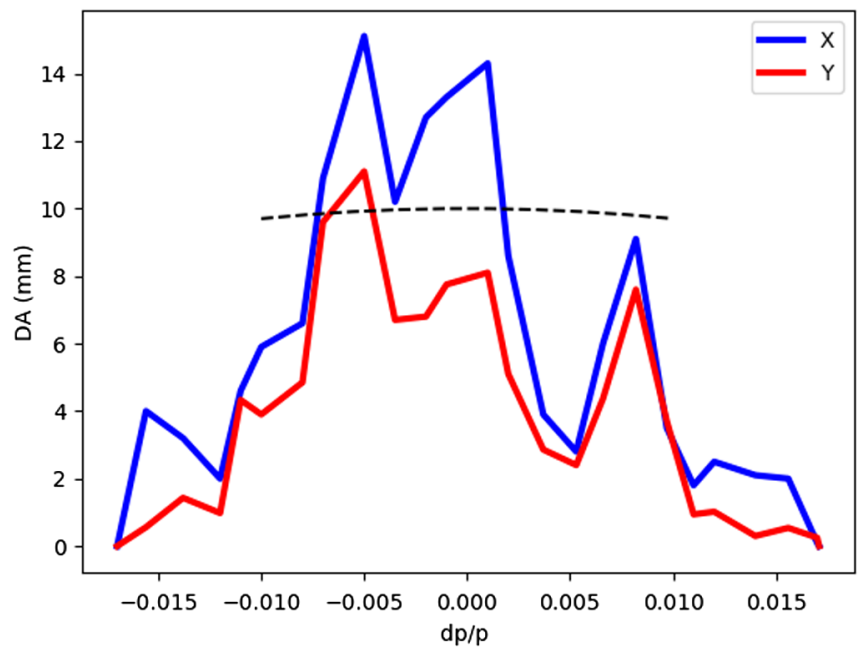

FIG. 13. Dynamic aperture vs momentum offset, $\beta_{x}=50 \mathrm{~m}$, $\beta_{y}=50 \mathrm{~m}$. Limitation due to the path lengthening effect for a typical rf setting is shown as a dashed line. 


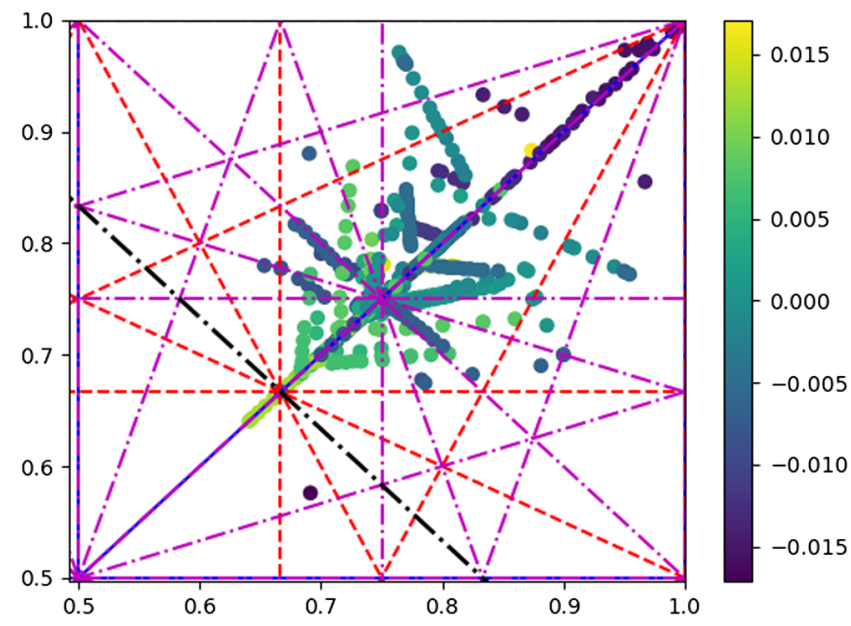

FIG. 14. Tune diagram for horizontal, vertical, and longitudinal degrees of freedom, color coded by momentum offset.

\section{CONCLUSION}

We presented a conceptual design of a noninterleaved round beam lattice for electron light sources. Its dynamic aperture and momentum acceptance have been analyzed. It is easy to implement in case round beam operation is the preferred mode. The dynamic aperture of the lattice is large, and is limited by the synchrobetatron coupling driven by the path lengthening effect. The momentum acceptance is somewhat restrictive, a situation common for noninterleaved lattices, but can be to a certain extent controlled by redistributing the chromaticity correction between ring octants. This type of lattice can potentially be used for other types of electron storage rings such as lepton colliders.

[1] A. Piwinski, Intra-beam scattering, in Proceedings of the 9th International Conference on High Energy Accelerators (HEACC 1974) (A.E.C., Washington, DC, 1975).

[2] J. Bjorken and S. Mtingwa, Intrabeam scattering, Part. Accel. 13, 115 (1983).

[3] K.-J. Kim, Optical and power characteristics of synchrotron radiation sources, Opt. Eng. (Bellingham, Wash.) 34, 342 (1995).

[4] P. Kuske, Round beam related challenges in storage ring light sources, in Proceedings of the Future Light Source 2018 Workshop, Shanghai, China, 2018 (JACoW, Shanghai, China, 2018).

[5] G. Guignard, Betatron coupling and related impact of radiation, Phys. Rev. E 51, 6104 (1995).

[6] D. A. Edwards and L. C. Teng, Parametrization of coupled motion in periodic systems, IEEE Trans. Nucl. Sci. 20, 885 (1973).

[7] D. Sagan and D. Rubin, Linear analysis of coupled lattices, Phys. Rev. ST Accel. Beams 2, 074001 (1999).

[8] I. Borchardt, E. Karantzoulis, H. Mais, and G. Ripken, Calculation of beam envelopes in storage rings and transport systems in the presence of transverse space charge effects and coupling, DESY Report No. 87-161, 1987.

[9] V.A. Lebedev and S. A. Bogacz, Betatron motion with coupling of horizontal and vertical degrees of freedom, Report No. FERMILAB-PUB-10-383-AD, 2012.

[10] R. Talman, A Proposed Möbius Accelerator, Phys. Rev. Lett. 74, 1590 (1995).

[11] A. N. Filippov et al., Proposal of the round beam lattice for VEPP-2M collider, in Proceedings of the 15th International Conference on High Energy Accelerators, Hamburg, Germany, 1992, p. 1145, https://cds.cern.ch/ record/245591.

[12] M. Aiba, M. Ehrlichman, and A. Streun, Round beam option in electron storage rings and generalization of Möbius accelerator, in Proceedings of IPAC2015, Richmond, VA, 2015 (JACoW, Richmond, USA, 2015).

[13] V. V. Danilov et al., The concept of round colliding beams, in Proceedings of EPAC96, Barcelona, 1996 (CRC Press, Boca Raton, USA, 1996), p. 1149.

[14] S. Hendersen et al., Investigation of the Möbius accelerator at CESR, in Proceedings of IPAC 1999, New York (JACoW, New York, USA, 1999).

[15] I. A. Koop, VEPP-2000 project, arXiv:physics/0106013.

[16] Y. Cai, K. Bane, R. Hettel, Y. Nosochkov, M.-H. Wang, and M. Borland, Ultimate storage ring based on fourthorder geometric achromats, Phys. Rev. ST Accel. Beams 15, 054002 (2012).

[17] L. Farvacque et al., A low-emittance lattice for the ESRF, in Proceedings of the 4th International Particle Accelerator Conference, IPAC-2013, Shanghai, China, 2013 (JACoW, Shanghai, China, 2013).

[18] M. Eriksson et al., Commissioning of the MAX IV light source, in Proceedings of IPAC'16, Busan, Korea, 2016 (JACoW, Busan, Korea, 2016).

[19] S. Henderson, Status of the APS upgrade project, in Proceedings of IPAC'15, Richmond, VA, 2015 (JACoW, Richmond, USA, 2015).

[20] D. Einfeld, J. Schaper, and M. Plesko, A pure insertion device synchrotron light source utilizing the MBA-optics, J. Phys. IV (France) 04, C9-373 (1994).

[21] D. Einfeld, M. Plesko, and J. Schaper, First multibend achromat lattice consideration, J. Synchrotron Radiat. 21, 856 (2014).

[22] L.C. Teng, Minimizing the emittance in designing the lattice of an electron storage ring, Fermilab Report No. TM-1269, 1984.

[23] C.X. Wang, Minimum emittance in storage rings with uniform or nonuniform dipoles, Phys. Rev. ST Accel. Beams 12, 061001 (2009).

[24] S. Y. Lee, Emittance optimization in three- and multiplebend achromats, Phys. Rev. E 54, 1940 (1996).

[25] K. Oide and H. Koiso, Dynamic aperture of electron storage rings with noninterleaved sextupoles, Phys. Rev. E 47, 2010 (1993).

[26] K. Brown, A second-order magnetic optical achromat, IEEE Trans. Nucl. Sci. 26, 3490 (1979).

[27] K. Balewski, W. Brefeld, W. Decking, H. Franz, R. Roehlsberger, and E. Weckert, Petra III: A low emittance synchrotron radiation source, DESY Technical Design Report, 2004. 
[28] W. Drube, M. Bieler, W. A. Caliebe, H. SchulteSchrepping, J. Spengler, M. Tischer, and R. Wanzenberg, The PETRA III extension, AIP Conf. Proc. 1741, 020035 (2016).

[29] R. Wanzenberg et al., Research activities towards a conversion of PETRA III into a diffraction limited synchrotron light source, in Proceedings of IPAC 2017, Copenhagen, Denmark (JACoW, Copenhagen, Denmark, 2017).

[30] D. Einfeld et al., A lattice design to reach the theoretical minimum emittance for a storage ring, in Proceedings of EPAC 1996, Barcelona, Spain (CRC Press, Boca Raton, USA, 1996).

[31] Y. Jiao et al., Parameter scaling and practical design of TME lattice, Report No. SLACPUB-14205, 2010.

[32] S. Y. Lee, Accelerator Physics, 3rd ed. (World Scientific, Singapore, 2012).

[33] M. Sands, The physics of electron storage rings: An introduction, Report No. SLAC-R-121, 1970.
[34] J. Bengtsson, The sextupole scheme for the Swiss Light Source (SLS): An analytic approach, SLS Note Report No. 9/97, 1997.

[35] E. Forest et al., Introduction to the polymorphic tracking code: Fiber bundles, polymorphic Taylor types and "Exact tracking", KEK Report No. 20023, 2002.

[36] F. Schmidt, MAD-X PTC integration, in Proceedings of the 21st Particle Accelerator Conference, Knoxville, TN, 2005 (IEEE, Piscataway, NJ, 2005).

[37] M. Hénon and C. Heiles, The applicability of the third integral of motion: Some numerical experiments, Astron. J. 69, 73 (1964).

[38] L. Emery, Coupling of betatron motion to the longitudinal plane through path lengthening in low alpha(c) storage rings, Int. J. Mod. Phys. Proc. Suppl. A 2B, 1172 (1993).

[39] Y. Shoji, Dependence of average path length betatron motion in a storage ring, Phys. Rev. ST Accel. Beams 8, 094001 (2005). 